\title{
A prototype for convective margin shifts
}

\author{
B. R. Lintner ${ }^{1}$ and J. D. Neelin ${ }^{1}$ \\ Received 20 June 2006; revised 6 November 2006; accepted 9 January 2007; published 10 March 2007.
}

[1] Regional precipitation anomalies under El Niño or global warming scenarios manifest considerable spatial variability, especially in the vicinity of tropical deep convective zones. Using a simplified set of equations, we develop an analytic prototype of convective margins for tropical land regions subject to inflow from adjacent ocean regions. The approach yields an expression for the location of the convective margin from which it is straightforward to deduce how climate perturbations impact the margin. We compare the results of the prototype to full model simulations of the equatorial South American precipitation response to El Niño forcing and find general agreement. Analysis of the observations supports the convective inflow margin prototype's applicability to appropriately simple basic state configurations. Citation: Lintner, B. R., and J. D. Neelin (2007), A prototype for convective margin shifts, Geophys. Res. Lett., 34, L05812, doi:10.1029/2006GL027305.

\section{Introduction}

[2] The gross-scale climate signatures of El Niño outside of the Pacific (the "remote teleconnection") include tropospheric and surface warming and widespread deficits in rainfall [Ropelewski and Halpert, 1987; Wallace et al., 1998]. While many studies have explored the mechanisms underlying these gross-scale responses [e.g., Klein et al., 1999; Saravanan and Chang, 2000; Chiang and Sobel, 2002; Su and Neelin, 2002; Chiang and Lintner, 2005; Lintner and Chiang, 2005; Neelin and Su, 2005], the regional scale features of the teleconnection are less well understood. For example, observed El Niño-related tropical precipitation anomalies exhibit a highly nontrivial spatial structure, with significant regional differences in the magnitudes and even sign of the anomalies [Dai and Wigley, 2000].

[3] The complex regional behavior points to the influence of multiple mechanisms on tropical precipitation variability [Neelin and Su, 2005]. For example, Neelin et al. [2003] and Chou and Neelin [2004] proposed a set of two related mechanisms-"upped-ante" and "rich-get-richer"-that are conceptually useful in the interpretation of the spatial structure of precipitation anomalies under El Niño or global warming forcing. Briefly, upped-ante postulates preferential moistening of convective zones relative to nonconvecting zones as warming increases the moisture threshold necessary to sustain convection, while the rich-get-richer mechanism suggests that the anomalous convective-nonconvective moisture gradient enhances convective region moisture convergence. While these mechanisms provide a template

\footnotetext{
${ }^{1}$ Department of Atmospheric and Oceanic Sciences and Institute of Geophysics and Planetary Physics, University of California, Los Angeles, California, USA.
}

Copyright 2007 by the American Geophysical Union. 0094-8276/07/2006GL027305\$05.00 for precipitation anomaly spatial structures-i.e., moistening of convective centers and drying along convective margins - their manifestation depends sensitively on factors such as the intensity and location of climatological convection and the relationship between winds and moisture gradients.

[4] From a modeling perspective, complexities in the spatial characteristics of tropical precipitation variability pose additional challenges. Simulations of El Niño show large intermodel discrepancies in the distribution and amplitude of precipitation changes [Joseph and Nigam, 2005]. Considerable intermodel disagreement is also evident in global warming simulations of precipitation, especially near strongly convecting regions in the tropics [Neelin et al., 2006]. Understanding why different models simulate the precipitation changes that they do is thus crucial to the use of models as predictive or interpretative tools.

[5] Our principal interest here is to understand the behavior of the tropical convective margin, i.e., the boundary separating convecting and nonconvecting regions, for the specific case of land region moisture inflow from a neighboring ocean region. We develop a prototype for the position of inflow margins and how margin locations vary under climate perturbations. Given the large discrepancies between models, the simplicity of the solution presented here is useful for developing intuition about a particular set of interactions contributing to precipitation anomalies under climate change scenarios.

\section{Model of the Convective Margin}

\subsection{Simplification of the Thermodynamic Equations}

[6] We start from vertically-averaged temperature and moisture equations, as in the work of Neelin and Zeng [2000] (see also the auxiliary material), and apply them, in steady-state and 1D, to a semi-infinite land region lying to the west of an ocean region (Figure 1). ${ }^{1}$ We assume evaporation to be small and therefore ignore it in the moisture equation. Precipitation is modeled as $P=\tau_{c}^{-1}(q-$ $q_{c}(T)$ ), where $q$ is vertically-averaged moisture, $q_{c}(T)$ is a vertically-averaged quasi-equilibrium (QE) value of moisture as a function of vertically-averaged tropospheric temperature, $T$, and $\tau_{c}$ is a convective-adjustment timescale. For $q<q_{c}(T), P=0$.

[7] Given these assumptions, the land region thermodynamic equations become:

$$
\begin{gathered}
M_{s} \nabla \cdot \mathbf{v}=P+F_{T} \\
-\left(M_{q p} q\right) \nabla \cdot \mathbf{v}=-u_{q} \partial_{x} q-P
\end{gathered}
$$

\footnotetext{
${ }^{1}$ Auxiliary materials are available in the HTML. doi:10.1029/ 2006 GL027305.
} 


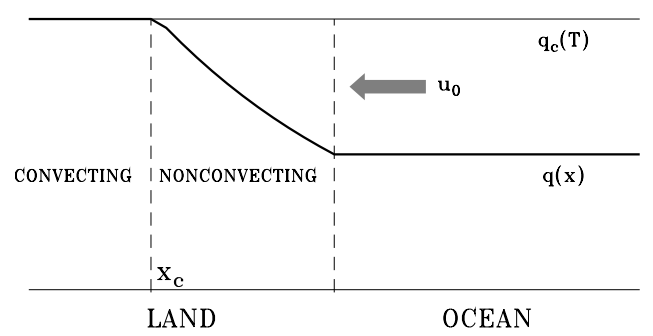

Figure 1. Schematic overview of the convective margins analysis. Here, $x_{c}$ denotes the position of the convective margin as defined by equation (3), relative to the coast, with inflow from the ocean.

where $F_{T}$ is the total flux forcing of the atmosphere, $u_{q}$ is the projection of the zonal wind field onto the vertical structure of moisture, and $M_{s}$ and $M_{q}$ are dry static stability and moisture stratification, respectively. Since the net surface flux vanishes in equilibrium, $F_{T}$ is just the net top-of-the atmosphere radiative forcing. We take $F_{T}>0$, which is appropriate for tropical or summertime continents [Chou and Neelin, 2003]. $M_{s}$ is assumed to be a spatial constant, though with distinct values in the convecting and nonconvecting regions, while $M_{q}$ is expressed as $M_{q p} q$. Note that for $T$ and $q$ in temperature units (e.g., K), $P$ and $F_{T}$ are in units of $\mathrm{K} \mathrm{s}^{-1} ; M_{s}$ and $M_{q}$ are also in $\mathrm{K}$.

[8] An additional approximation, that of weak temperature gradients (WTG), has been utilized in equation (1) [Bretherton and Sobel, 2002; Sobel et al., 2002]. WTG follows from the observed tendency for gradients in $T$ to be small within the Tropics, as a result of the efficient lateral homogenization of temperature by equatorial wave dynamics. As such, advective $T$ gradients in equation (1) are neglected, as are any temperature-related spatial dependences of the flux terms. The utility of WTG is that, for specified $T, \nabla \cdot \mathbf{v}$ is determined by equation (1) and can be substituted into equation (2). Ocean region $q$ is assumed given, with $q_{0}$ representing the boundary value for land region moisture.

\subsection{Solution for the Nonconvecting Region}

[9] Integrating equation (2) westward from the boundary (at $x=0$ ) for the case of $u_{q}=$ constant yields $q(x)=q_{0} e^{-\lambda x}$, for all $x$ such that $q<q_{c}(T)$, where the length-scale $\lambda^{-1}$ is given by $-u_{q} M_{s}\left(M_{q p} F_{T}\right)^{-1}$. The position of the convective margin relative to the land-ocean interface, $x_{c}$, is then given by the value of $x$ for which $q=q_{c}(T)$, i.e.,:

$$
x_{c}=-\lambda^{-1} \ln \left(q_{c}(T) / q_{0}\right)
$$

\subsection{Solution for the Convecting Region}

[10] Within the convecting region (i.e., $x \leq x_{c}$ ), QE constrains $q$ to be close to $q_{c}(T)$, so $M_{q} \approx M_{q p} q_{c}(T)$. Eliminating $\nabla \cdot \mathbf{v}$ between equations (1) and (2) and using the definition of $P$ leads to a first order differential equation with solution $q(x)=q_{c}(T)+\tau_{c} M_{q p} q_{c}(T) F_{T} M^{-1}(1-$ $\left.e^{\lambda c(x-x c)}\right)$, where the gross moist stability $M=M_{s}-M_{q}$ [Yu et al., 1998] is approximately constant. $\lambda_{c}^{-1} \stackrel{M^{\prime}}{=}$ $-u_{q} \tau_{c} M_{s} M^{-1}$ defines a characteristic length-scale over which $q$ transitions to its maximum value. The precipitation field is thus:

$$
P(x)=\frac{M_{q p} q_{c}(T)}{M} F_{T}\left(1-e^{\lambda_{c}\left(x-x_{c}\right)}\right)
$$

[11] In the "strict-QE limit" of $\tau_{c} \rightarrow 0, P=M_{q p} q_{c}(T)$ $F_{T} M^{-1}$, i.e., the precipitation field transitions abruptly from 0 to its peak value at the margin $x_{c}$.

\subsection{Perturbations of the Convective Margin}

[12] Our primary interest in developing the convective margin model is to understand what happens to the convective margin under anomalous climate forcing (e.g., El Niño or global warming). Consider a $T$ increase $\delta T$. According to equation (3), the location of the margin $x_{c}$ recedes from the coastline, since $q_{c}(T)$ increases. Precisely how far the margin moves depends on how far the inflow value of moisture $q_{0}$ is from the threshold for deep convection, the (low-level) wind, and the thermodynamic dependences of $\lambda^{-1}$.

[13] What happens to the precipitation field in the warming scenario? Consider an anomalous marginal displacement $\delta x_{c}$ associated with positive $\delta T$. For $x<x_{c}+\delta x_{c}$, the convective region precipitation increases by $\delta P=M_{q p}$ $\left(d q_{c}(T) / d T\right) \delta T F_{T} M^{-1}$, while for $x>x_{c}, \delta P=0$. For the region located within the anomalous shift zone, $x_{c}+\delta x_{c}<$ $x<x_{c}$, anomalous displacement of the convective margin completely shuts down the mean precipitation. Thus, the inflow margin shift produces strong droughts in those regions near the edge of the convective zone that no longer support convection.

[14] The convective margin is modeled here as a discontinuity. On timescales of individual convective events, a sharp convective margin is not unreasonable. However, for longer timescales (such as monthly averages), the edge of convection tapers smoothly, so that identification of a distinct convective margin is challenging. Equation (3) provides insight into smooth edge of the convective zone. For example, consider a wind field consisting of a steady component $\bar{u}_{q}$ and a time-varying, Gaussian perturbation $\delta u_{q}$ with standard deviation $\sigma_{\delta_{u_{q}}}$. Then, $x_{c}=\bar{x}_{c}\left(1+\delta u_{q} \bar{u}_{q}^{-1}\right)=\bar{x}_{c}+$ $\delta x_{c}$. Keeping all other parameters fixed, the spatial variation of $P$ associated with an ensemble of such perturbations is

$$
P(x)=\frac{P_{0}}{2 \sigma_{\delta x_{c}} \sqrt{(\pi)}} \int_{x-\bar{x}_{c}}^{\infty} e^{\frac{-\delta x_{x^{2}}}{2 \sigma_{x_{c}}^{2}}} d\left(\delta x_{c}\right)
$$

[15] $P(x)$ transitions between $P_{0}$ and 0 over a characteristic width $2 \sqrt{2} \sigma_{\delta_{u_{q}}}\left|\bar{x}_{c} / \bar{u}_{q}\right|$ centered on $\bar{x}_{c}$, so wind field transients smooth the convective margin (Figure 2a). Perturbations to these more realistic profiles occur on the scale of the mean displacement of $x_{c}$ (Figure 2b).

\section{Convective Margin Shifts in El Niño Simulations}

[16] To illustrate the utility of the margins prototype, we consider the impact of El Niño on the location of the convective margin, with a regional focus on equatorial South America. Our emphasis on this region follows from the substantial El Niño-related precipitation anomalies that 

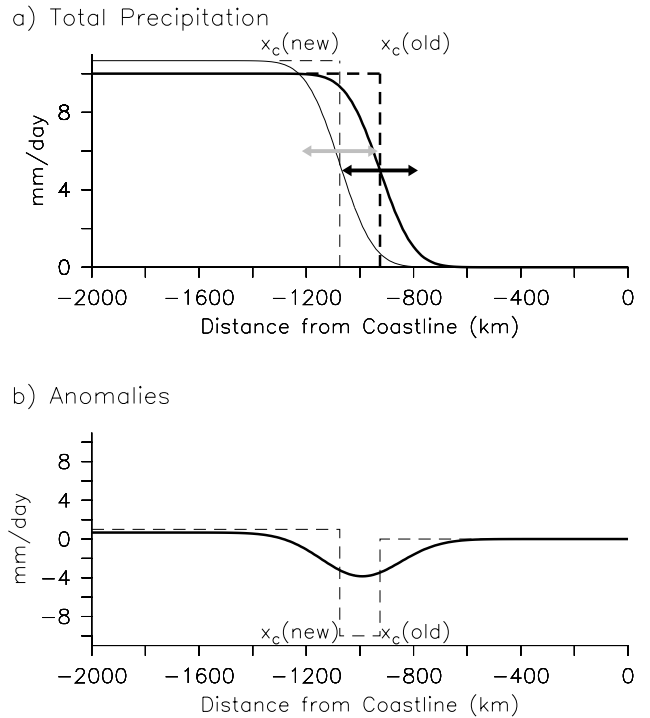

Figure 2. Perturbations to the convective margin. (a) Idealized examples of convective margins subject to stochastic perturbations (equation (5)). The profiles reflect peak precipitation values of 10 and $11 \mathrm{~mm}$ day $^{-1}$ and margin locations (relative to the coast) of $925 \mathrm{~km}$ and $1075 \mathrm{~km}$; the profile with the higher peak precipitation rate and greater margin distance from the coast corresponds to a $1 \mathrm{~K}$ temperature perturbation relative to the lower precipitation rate/smaller margin location profile. Included in both profiles is a stochastic perturbation of $2 \mathrm{~m} \mathrm{~s}^{-1}$, or $1 / 2$ the mean $\bar{u}_{q}$ shows (b) the difference between the two Gaussian-perturbed profiles in Figure 1a (solid line); also shown is the difference for margins without stochastic effects (dashed line).

occur there in both the observations and simulations [e.g., Hastenrath et al., 1987; Joseph and Nigam, 2005]. Mean-state easterly advection of moisture $\left(u_{q}<0\right)$ is significant in this region because of near-equatorial tradewind convergence.

[17] We compare $x_{c}$ predicted by equation (3) to estimates of the convective margin location derived from a 10-member ensemble of 51-year integrations of the Quasi-equilibrium Tropical Circulation Model 1 version 2.3 (QTCM1) [Neelin and Zeng, 2000; Zeng et al., 2000]. Each ensemble member was initialized from a set of independent initial conditions and integrated over the period 1950-2000, with observed sea surface temperatures (SSTs) specified over the tropical Pacific and a passive, thermal ocean mixed layer of $50 \mathrm{~m}$ depth applied elsewhere. Over tropical South America, the mean precipitation increases (at least in certain seasons) away from the Atlantic coast to a maximum within the interior of the continent. Estimates of the simulated convective margin were obtained by first determining the longitude, relative to the Atlantic coastline, at which the precipitation field equals half of its maximum climatological value over South America at each latitude, and then averaging over $10^{\circ} \mathrm{S}-0^{\circ}$.

[18] For September-October-November (SON), the mean estimated position of the convective margin as defined here is $\sim 930 \mathrm{~km}$ from the Atlantic coastline of South America. Comparison of the margin estimates to NINO3, an index of ENSO variability consisting of SSTs averaged over $150^{\circ} \mathrm{W}-90^{\circ} \mathrm{W}$ and $5^{\circ} \mathrm{S}-5^{\circ} \mathrm{N}$, indicates that the convective margin retreats further away from the coast when NINO3 region SSTs are anomalously warm (Figure 3, triangles). That is, El Niño conditions are associated with a broader nonconvecting region, and thus drier-than-normal conditions, over eastern tropical South America. Progressive westward shifting of the convective margin may explain the increase in the areal extent of tropical droughts with increasing El Niño strength [Lyon, 2004; Lyon and Barnston, 2005].

[19] The $x_{c}$ values predicted by the margin prototype match the estimates derived from the QTCM1 simulations rather well (Figure 3, squares). Using equation (3), one can further decompose the impact of ENSO forcing on the convective margin by considering how each factor responds to the forcing. For the ensemble-averaged QTCM1 results, the westward displacements of $x_{c}$ with above normal NINO3 SSTs are principally dominated by the increase to the convective threshold $q_{c}(T)$ as the troposphere warms. Of course, $u_{q}$ also becomes more negative (i.e., more easterly) under El Niño conditions and thus enhances the westward displacement of $x_{c}$ over South America. On the other hand, $q_{0}$ tends to increase with positive NINO3 index (since the adjacent tropical Atlantic warms), thereby partially offsetting the westward shifts associated with larger $q_{c}(T)$ and anomalous easterlies. It should be noted that the relationship between NINO3 and convective margin location holds for individual ensemble members, although there is greater scatter associated with atmospheric interval variability.

\section{Applicability to the Observations}

[20] A crucial step in assessing the applicability of the margin prototype to the observations is the identification of

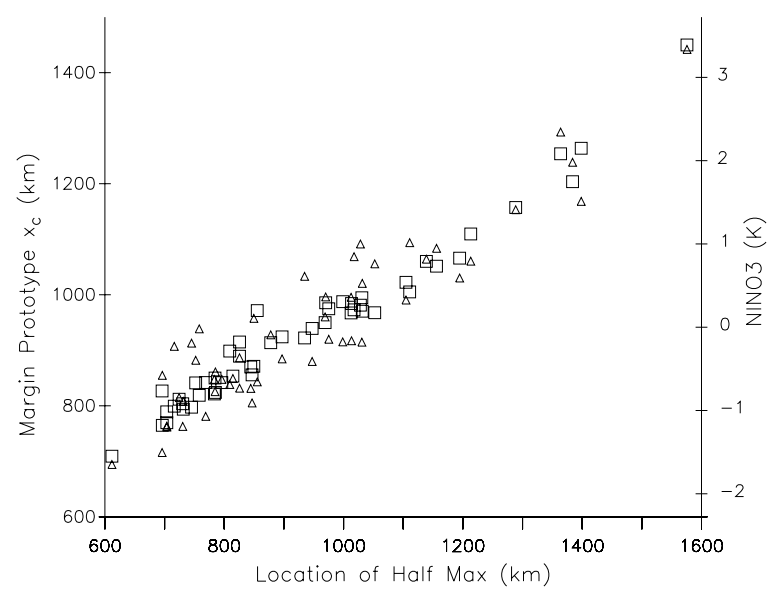

Figure 3. Convective margin position variations over equatorial South America from QTCM1 simulations. Simulated margin locations ( $\mathrm{x}$-axis; in $\mathrm{km}$ ) were estimated from the longitude at which the total precipitation, averaged over September-October-November $(\mathrm{SON})$ and $10^{\circ} \mathrm{S}-0^{\circ}$, achieved half its maximum climatological value in the interval $70^{\circ} \mathrm{W}-30^{\circ} \mathrm{W}$. The longitude values were converted to distances relative to the mean South American Atlantic coastal margin longitude $\left(41^{\circ} \mathrm{W}\right)$ between $10^{\circ} \mathrm{S}-0^{\circ} \mathrm{S}$. Plotted against these estimates are values of the NINO3 index (in $\mathrm{K}$; triangles) as well as margin prototype $x_{c}$ predicted by equation (3) (in km; squares). 


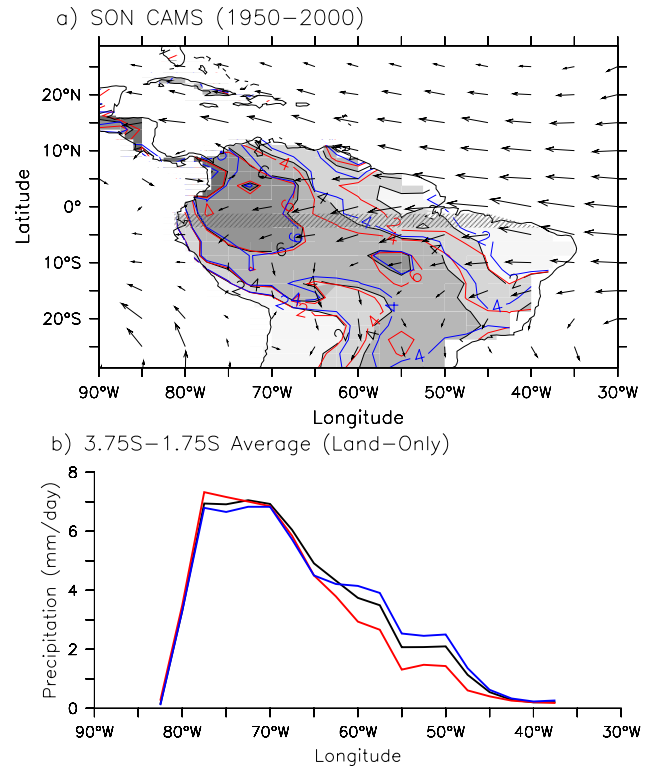

Figure 4. SON composites of observed CAMS rain gauge precipitation for South America during 1950-2000. (a) El Niño phase average (red contours) consists of those years for which the SON-averaged NINO3 $\geq 1$ standard deviation, while the La Niña phase (blue contours) consists of those years with NINO3 $\leq-1$ standard deviation. Also shown are the mean SON precipitation over all years (black contours, shaded for emphasis) and the mean $850 \mathrm{mb}$ wind field (vectors) from the NCAR/NCEP 40-year Reanalysis. (b) Longitudinal transects, averaged over the stippled area in Figure 4a for El Niño (red), La Niña (blue), and all years (black).

circumstances under which the basic assumptions hold. Although we anticipate that the physics should apply more generally, the simple 1D prototype developed here can be applied only when the geometries of both the low-level wind and precipitation fields are relatively uncomplicated. As suggested by the observed Climate Anomaly Monitoring System (CAMS) rain gauge-derived precipitation [Chen et al., 2002] and NCAR/NCEP 40-year Reanalysis $850 \mathrm{mb}$ wind field [Kalnay et al., 1996] over tropical South America, the SON season appears well suited to use of the prototype, since mean low-level circulation is essentially zonal and normal to the mean precipitation isolines (Figure 4a; see also the auxiliary material). Other caveats, such as fairly constant inflow moisture values along the coastal margin, are largely satisfied in this season as well.

[21] Compositing the SON CAMS rainfall with respect to the El Niño and La Niña phases of the SON-averaged NINO3 index shows behavior resembling the QTCM1 response to ENSO. In particular, under El Niño conditions, there is a general westward displacement of the precipitation isolines over much of near-equatorial South America (Figure 4a, red contours). Consider, for example, the $4 \mathrm{~mm}$ day $^{-1}$ contour, which generally parallels the Atlantic coastline of South America over $20^{\circ} \mathrm{S}-10^{\circ} \mathrm{N}$ : the zonal position of this contour under El Niño conditions is shifted west of its mean location by up to $5^{\circ}$.

[22] To obtain a more quantitative picture of the changes to the structure of the precipitation field under El Niño and
La Niña conditions, we averaged the composites over $3.8^{\circ} \mathrm{S}-1.2^{\circ} \mathrm{S}$; results of this averaging appear in Figure $4 \mathrm{~b}$. The most pronounced differences between the El Niño (red line) and La Niña (blue line) longitudinal profiles occur between $50^{\circ} \mathrm{W}-60^{\circ} \mathrm{W}$, i.e., at mean precipitation values roughly $1 / 3$ to $1 / 2$ the peak SON rainfall values. Relatively small differences prevail where the precipitation rates are highest. These characteristics are broadly consistent with those shown schematically in Figure 2, indicating that the notion of viewing the observed precipitation changes in this region as a convective margin shift has some merit. An assessment of the factors contributing to the observed margin displacements will be undertaken in future work.

\section{Summary and Discussion}

[23] We present an analytic prototype of land region convective margins for the case of moisture inflow from a neighboring ocean region. A simple functional expression is obtained for the position of the convective margin. This expression facilitates diagnosis of precipitation changes occurring along tropical land region convective margins under climate perturbation forcing scenarios. For example, comparisons of the simple analytic results to simulations of ENSO-related precipitation impacts over tropical South America demonstrate reasonable agreement. Observed precipitation anomalies under certain conditions also appear consistent with the margin prototype.

[24] The convective inflow margin prototype succinctly illustrates (1) the utility of viewing certain regional precipitation anomalies as shifts in the position of the convective margin; and (2) the factors affecting such shifts over tropical land regions, including the dependence of the convective threshold on tropospheric temperature and the inflow wind and moisture values. Although we emphasize here a specific regional impact of El Niño forcing, the approach is applicable to other tropical land regions as well as other forcing scenarios. For example, we hope to use the convective margins theory as a template for analyzing regional changes occurring under greenhouse gas warming scenarios as simulated by different models.

[25] Acknowledgments. Financial support for this work was provided by NOAA grants NA05OAR4311134 and NA05OAR4310007 and NSF grants ATM-0082529 and ATM-0645200. BRL also acknowledges partial support of NOAA grant NA03OAR4310066 (John C. H. Chiang, UC Berkeley).

\section{References}

Bretherton, C. S., and A. H. Sobel (2002), A simple model of a convectively-coupled Walker circulation using the weak temperature gradient approximation, J. Clim., 15, 2907-2920.

Chen, M., P. P. Xie, J. E. Janowiak, and P. A. Arkin (2002), Global land precipitation: A 50-year monthly analysis based on gauge observations, J. Hydrometeor., 3, 249-266.

Chiang, J. C. H., and B. R. Lintner (2005), Mechanisms of remote tropical surface warming during El Niño, J. Clim., 18, 4130-4149.

Chiang, J. C. H., and A. H. Sobel (2002), Tropical tropospheric temperature variations caused by ENSO and their influence on the remote tropical climate, J. Clim., 15, 2616-2631.

Chou, C., and J. D. Neelin (2003), Mechanisms limiting the northward extent of the northern summer monsoons over North America, Asia, and Africa, J. Clim., 16, 406-425.

Chou, C., and J. D. Neelin (2004), Mechanisms of global warming impacts on regional tropical precipitation, J. Clim., 17, 2688-2701. 
Dai, A., and T. M. L. Wigley (2000), Global patterns of ENSO-induced precipitation, Geophys. Res. Lett., 27, 1283-1286.

Hastenrath, S. L., C. De Castro, and P. Aceituno (1987), The Southern Oscillation in the tropical Atlantic sector, Contrib. Atmos. Phys., 60, $447-463$.

Joseph, R., and S. Nigam (2005), ENSO evolution and teleconnections in IPCC's 20th century climate simulations: Realistic representation? J. Clim., 19, 4360-4377.

Kalnay, E., et al. (1996), The NCEP/NCAR 40-Year Reanalysis Project, Bull. Am. Meteorol. Soc., 77, 437-471.

Klein, S. A., B. J. Soden, and N. C. Lau (1999), Remote sea surface temperature variations during ENSO: Evidence for a tropical atmospheric bridge, J. Clim., 12, 917-932.

Lintner, B. R., and J. C. H. Chiang (2005), Reorganization of tropical climate during El Niño: A weak temperature gradient approach, J. Clim., $18,5312-5329$.

Lyon, B. (2004), The strength of El Niño and the spatial extent of tropical drought, Geophys. Res. Lett., 31, L21204, doi:10.1029/2004GL020901.

Lyon, B., and A. G. Barnston (2005), ENSO and the spatial extent of interannual precipitation extremes in tropical land areas, J. Clim., 18, $5095-5109$.

Neelin, J. D., and H. Su (2005), Moist teleconnection mechanisms for the tropical South American and Atlantic sector, J. Clim., 18, 3928-3950.

Neelin, J. D., and N. Zeng (2000), A quasi-equilibrium tropical circulation model-Formulation, J. Atmos. Sci., 57, 1741-1766.

Neelin, J. D., C. Chou, and H. Su (2003), Tropical drought regions in global warming and El Niño teleconnections, Geophys. Res. Lett., 30(24), 2275 , doi:10.1029/2003GL018625.
Neelin, J. D., M. Munnich, H. Su, J. E. Meyerson, and C. E. Holloway (2006), Tropical drying trends in global warming models and observations, Proc. Natl. Acad. Sci. U. S. A., 103, 6110-6115.

Ropelewski, C. F., and M. S. Halpert (1987), Global and regional scale precipitation patterns associated with the El Niño Southern Oscillation, Mon. Weather Rev., 115, 1606-1625.

Saravanan, R., and P. Chang (2000), Interaction between tropical Atlantic variability and El Niño Southern Oscillation, J. Clim., 13, 2177-2194.

Sobel, A. H., I. M. Held, and C. S. Bretherton (2002), The ENSO signal in tropical tropospheric temperature, J. Clim., 15, 2702-2706.

$\mathrm{Su}$, H., and J. D. Neelin (2002), Teleconnection mechanisms for tropical Pacific descent anomalies during El Niño, J. Atmos. Sci., 59, 2694-2712. Wallace, J. M., E. M. Rasmusson, T. P. Mitchell, V. E. Kousky, E. S. Sarachik, and H. von Storch (1998), The structure and evolution of ENSO-related climate variability in the tropical Pacific: Lesson from TOGA, J. Geophys. Res., 103, 14,241-14,259.

Yu, J.-Y., C. Chou, and J. D. Neelin (1998), Estimating the gross moist stability of the tropical atmosphere, J. Atmos. Sci., 55, 1354-1372.

Zeng, N., J. D. Neelin, and C. Chou (2000), A quasi-equilibrium tropical circulation model-Implementation and simulation, J. Atmos. Sci., 57, $1767-1796$

B. R. Lintner and J. D. Neelin, Department of Atmospheric and Oceanic Sciences and Institute of Geophysics and Planetary Physics, University of California Los Angeles, Los Angeles, CA 90095-1565, USA. (ben@atmos. ulca.edu; neelin@atmos.ucla.edu) 